\section{Reduced-complexity transmit-beamforming codebook search algorithm}

\section{Y.J. Kim, S.H. Won, N.Y. Park and L. Hanzo}

A two-stage reduced-complexity index search algorithm is proposed for finding the best vector in the codebook of quantised equal gain transmission based multiple-input multiple-output arrangements When the number of transmit antennas is more than three, the normalised complexity is halved while maintaining the same symbol error rate as the benchmark.

Introduction: The de facto fourth generation $(4 \mathrm{G})$ wireless systems have encompassed codebook (CB) based closed-loop multiple-input multiple-output (MIMOs) [1]. Quantised equal gain transmission (QEGT) imposes less stringent transmit amplifier linearity requirements than other MIMOs. Similar to other CB based MIMO schemes, QEGT requires a substantially increased $\mathrm{CB}$ size, as the number of transmit antennas increases. Hence, employing an efficient $\mathrm{CB}$ index search algorithm becomes essential. A single-user MIMO-OFDM system relying on transmit beamforming (BF) and using $M_{t}$ transmit as well as $M_{r}$ receive antennas is considered. The system model and QEGT $\mathrm{CB}$ design are the same as in [2]. This allows the direct contrasting of our efficient search to that in [2]. The matrix of channel impulse responses (CIR) $\boldsymbol{H} \in \mathbb{C}^{M_{r} \times M_{t}}$ is assumed to have IID complex-valued Gaussian elements having a unit variance. The CIR is assumed to be perfectly estimated at the downlink receiver. Given the transmitted symbol of rank one $s \in \mathbb{C}$, the received symbol $x \in \mathbb{C}$ is described by

$$
x=z^{H} \boldsymbol{H w} s+z^{H} \boldsymbol{n}
$$

where $z^{H} \in \mathbb{C}^{1 \times M_{r}}$ is the combining vector, $\boldsymbol{w} \in \mathbb{C}^{M_{t} \times 1}$ is the BF vector having $\|\boldsymbol{w}\|=1$ and $\boldsymbol{n} \in \mathbb{C}^{M_{r} \times 1}$ represents the complex-valued AWGN having a variance of $N_{o}$. When the number of feedback bits is $L$, the CB size is denoted by $N=2^{L}$.

Two-stage index search algorithm: To obtain a fast index search for the QEGT CB, we propose to group the $\mathrm{BF}$ vectors in the $\mathrm{CB}$ into a given number of categories based on their chordal distance expressed by

$$
d\left(\boldsymbol{w}_{j}, \boldsymbol{w}_{k}\right)=\sqrt{1-\left|\boldsymbol{w}_{j}^{H} \boldsymbol{w}_{k}\right|^{2}}
$$

where $\boldsymbol{w}_{i}$ and $\boldsymbol{w}_{k}$ are arbitrary BF vectors in the CB. The BF vectors can be grouped by utilising a Euclidean space. This Letter applies Lloyd's algorithm to the $\mathrm{BF}$ vectors of the $\mathrm{CB}$ to generate their optimal grouping. To illustrate the grouping method, we consider the case of $\left(M_{t}, N\right)=(2$, 64). The first element of the BF vectors corresponding to the first transmit antenna can be forced to be real-valued owing to the rotation-invariant property of $\mathrm{BF}$ vectors [3]. Then, the second element of the BF vectors corresponding to the second transmit antenna can be plotted on the complex-valued plane, as seen in Fig. 1, which illustrates eight groups of $\mathrm{BF}$ vectors as the result of Lloyd's clustering algorithm. In the notation of $\boldsymbol{w}_{i, j}$ seen in Fig. 1, $i$ represents the $i$ th element of the $\mathrm{BF}$ vector corresponding to the $i$ th transmit antenna and $j$ denotes the index of the $\mathrm{BF}$ vector in the CB. Fig. 1 also magnifies a group of eight $\mathrm{BF}$ vectors at the right, where the cluster centroid is indicated by a square mark, while the others are indicated by triangles. In the same way, we can group the $\mathrm{BF}$ vectors in the $\mathrm{CB}$, when the number of transmit antennas is higher than or equal to three. Assuming that the QEGT $\mathrm{CB}$ elements are uniformly quantised, they can be arranged into $P$ groups, each having $Q \mathrm{BF}$ vectors. Therefore the $\mathrm{CB}$ size is $N=P Q$.

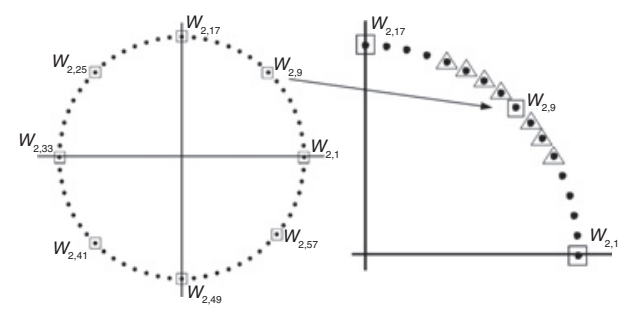

Fig. 1 Example of $C B$ index grouping when $\left(M_{t}, N\right)=(2,64)$

Now we are ready to apply our two-stage index search algorithm. In the first stage, the best of the centroids is selected, which maximises the channel gain of

$$
\boldsymbol{c}_{\text {opt }}=\arg \max _{\boldsymbol{c}_{p} \in C_{\text {set }}}\left\|\boldsymbol{H} \boldsymbol{c}_{p}\right\|
$$

where $\boldsymbol{c}_{p}, p=1,2, \ldots, P$ are the centroids, while $C_{\text {set }}$ is the set of $\left\{\boldsymbol{c}_{1}, \boldsymbol{c}_{2}\right.$, $\left.\ldots, c_{P}\right\}$. At the second stage, finally, the optimal $\mathrm{BF}$ vector is determined within the group of the selected centroid, which maximises the following equation:

$$
\boldsymbol{m}_{\text {opt }}=\arg \max _{\boldsymbol{m}_{q} \in M_{\text {set }}}\left\|\boldsymbol{H} \boldsymbol{m}_{q}\right\|
$$

where $\boldsymbol{m}_{q}, q=1,2, \ldots, Q$, represents the BF vectors of the chosen group and $M_{\text {set }}$ is the set of $\left\{\boldsymbol{m}_{1}, \boldsymbol{m}_{2}, \ldots, \boldsymbol{m}_{Q}\right\}$. As a benefit, the entire search complexity is significantly reduced. The problem now becomes how to determine the number of groups $P$ and the number of elements $Q$ in each group, when the CB size, $N$, is fixed. Based on our investigations not included here, the grouping rule can be applied that the number of groups $P$ should be the same as, or the nearest integer to, the value of $N / P$ when the $\mathrm{BF}$ vectors in the $\mathrm{CB}$ are distributed almost evenly to each group.

Simulation results: The CB search complexity and the average SER will be characterised for both the conventional and the proposed search. BF arrangements having three-to-five transmit antennas and two receive antennas are considered. QPSK modulation is assumed for the MIMO-OFDM LTE system, the carrier frequency of which is $2 \mathrm{GHz}$, system bandwidth is $5 \mathrm{MHz}$, sampling rate is $7.68 \mathrm{MHz}$, cyclic prefix size is 30 , FFT size is 512 , and the number of subcarriers occupied is set to 300 . The channel model is assumed to be the ITU-R PedestrianB model having six paths [4]. It is also assumed that the channel estimation and symbol synchronisation are ideal. There is no spatial correlation amongst the antennas. Table 1 quantifies how much the $\mathrm{CB}$ search complexity is reduced in comparison to the conventional search. When the number of groups $P$ is fixed, the number of vectors in each group may vary around the nearest integer of $N / P$. The number of indices searched, namely $P+\bar{Q}$ (where $\bar{Q}$ is the mean of $Q$ ) becomes the lowest among the other grouping configurations, when our grouping rule defined in the preceding Section is applied. The CB search complexity was also evaluated in real time based on a Texas Instruments TMS6713 DSP chip. The normalised complexity of the proposed technique is given by dividing the search complexity of the proposed method by the conventional one, as summarised in the last column of Table 1 . As the CB size $N$ increases, the normalised complex gain of the proposed scheme becomes more substantial.

\begin{tabular}{|c|c|c|c|c|c|}
\hline$M_{t}$ & $N=2^{L}$ & $P$ & $\bar{Q}$ & $\begin{array}{c}\text { Number of indices } \\
\text { searched/number of total } \\
\text { indices }\{(P+\bar{Q}) / N\}\end{array}$ & $\begin{array}{c}\text { Normalised complexity } \\
\text { of proposed search for } \\
\text { TI DSP }\end{array}$ \\
\hline \multirow{3}{*}{3} & \multirow{3}{*}{16} & 2 & 8 & $10 / 16=0.625$ & 0.628 \\
\hline & & 4 & 4 & $8 / 16=0.500$ & 0.503 \\
\hline & & 8 & 2 & $10 / 16=0.625$ & 0.628 \\
\hline \multirow{6}{*}{4} & \multirow{3}{*}{16} & 2 & 8 & $10 / 16=0.625$ & 0.628 \\
\hline & & 4 & 4 & $8 / 16=0.500$ & 0.503 \\
\hline & & 8 & 2 & $10 / 16=0.625$ & 0.628 \\
\hline & \multirow{3}{*}{64} & 4 & 16 & $20 / 64=0.313$ & 0.313 \\
\hline & & 8 & 8 & $16 / 64=0.250$ & 0.251 \\
\hline & & 16 & 4 & $20 / 64=0.313$ & 0.313 \\
\hline \multirow{6}{*}{5} & \multirow{3}{*}{16} & 2 & 8 & $10 / 16=0.625$ & 0.628 \\
\hline & & 4 & 4 & $8 / 16=0.500$ & 0.503 \\
\hline & & 8 & 2 & $10 / 16=0.625$ & 0.628 \\
\hline & \multirow{3}{*}{64} & 4 & 16 & $20 / 64=0.313$ & 0.313 \\
\hline & & 8 & 8 & $16 / 64=0.250$ & 0.251 \\
\hline & & 16 & 4 & $20 / 64=0.313$ & 0.313 \\
\hline
\end{tabular}

Table 1: Comparisons among number of average candidate weight vectors under two systems considered when assuming $M_{r}=1$

The SER performance of both the proposed schemes as well as of the conventional one is shown in Fig. 2. The number of transmit antennas is three to five and the number of receive antennas is set to two. When the value of $N$ is fixed to 16 or 64 , there are various grouping configurations. As the number of groups $P$ increases, the SER is improved at the cost of an increased CB search. When the two-stage grouping rule is applied, i.e. we have $(N, P)=(16,4)$ or $(64,8)$, the performance degradation 
becomes less than $0.2 \mathrm{~dB}$, while maintaining the lowest search complexity. Hence, an attractive trade-off was struck between the $\mathrm{CB}$ search complexity and SER performance.

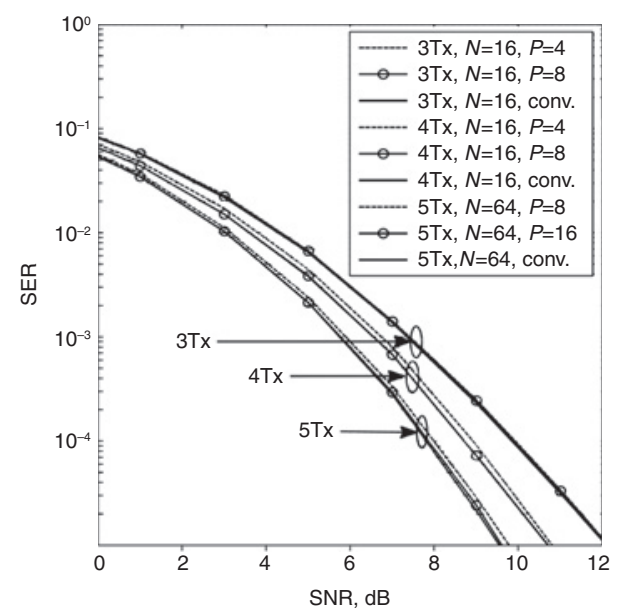

Fig. 2 SER performance of various grouping configurations when $\left(M_{t}, N\right)=$ $(3,16),(4,16)$ and $(5,64)$

Conclusion: A reduced-complexity closed-loop downlink BF scheme has been investigated, which relies on a grouping strategy and imposes only a slight SNR loss, when operating at half the complexity of the conventional search and relying on three-to-five transmit antennas.

Acknowledgment: This research was supported by the Basic Science Research Program through the National Research Foundation of Korea (NRF) funded by the Ministry of Education, Science and Technology (2010-0002702).

(C) The Institution of Engineering and Technology 2011

23 April 2011

doi: $10.1049 / \mathrm{el} .2011 .1202$

Y.J. Kim and N.Y. Park (Department of Information and Communication, Chungbuk National University, Republic of Korea)

E-mail: yjkim@cbnu.ac.kr

S.H. Won and L. Hanzo (School of ECS, University of Southampton, Southampton SO17 1BJ, United Kingdom)

\section{References}

1 Love, D.J., Heath, R.W., Lau, V.K.N., Rao, B.D., and Andrews, M.: 'An overview of limited feedback in wireless communication systems', IEEE J. Sel. Areas Commun., 2008, 26, (8), pp. 1341-1365

2 Love, D.J., and Heath, R.W.: 'Equal gain transmission in multiple-input multiple-output wireless systems', IEEE Trans. Commun., 2003, 51, (7), pp. $1102-1110$

3 Love, D.J., Heath, R.W., and Strohmer, T.: 'Grassmannian beamforming for multiple-input multiple-output wireless systems', IEEE Trans. Inf. Theory, 2003, 49, (10), pp. 2735-2747

4 Recommendation ITU-R M.1225: 'Guidelines for evaluation of radio transmission technologies for IMT-2000’, 1997 